Resumo de Tese

\title{
Interrupção médica da gestação de fetos com anomalias letais
}

\author{
Medical termination of pregnancy with lethally abnormal fetuses
}

Autora - Luciana Vivas Silva

Orientador - Prof. Dr. José Guilherme Cecatti

Dissertação de Mestrado apresentada ao Programa de Pós-Graduação da Faculdade de Ciências Médicas da Universidade Estadual de Campinas, para obtenção do Título de Mestre em Tocoginecologia (área de Tocoginecologia), 31 de agosto de 2006

Objetivos: avaliar comparativamente as mulheres que optaram pela interrupção médica da gestação por anomalias fetais incompativeis com a vida, no Serviço de Medicina Fetal do Centro de Atenção Integral à Saúde da Mulher (CAISM) da Universidade Estadual de Campinas (UNICAMP), quanto à realização do feticídio, considerando às características gerais e a evolução clínica. Sujeitos e Métodos: realizada investigação operacional comparando 146 mulheres de diversas idades gestacionais, acompanhadas no Ambulatório de Medicina Fetal do CAISM desde julho de 1994 a janeiro de 2006, que tiveram diagnóstico ultrasonográfico ou genético de anomalia fetal letal e optaram pela interrupção da gestação mediante autorização judicial. Os grupos I (submetido ao feticídio; $\mathrm{n}=82$ ) e II (indução de parto com feto vivo; $n=64$ ) foram comparados em relação às características gerais e aos aspectos clínicos. Para análise dos dados foram utilizados os testes de Fisher, $\mathrm{x}^{2}$ e a Regressão Logística de Cox, com significância de 0.05. Resultados: os grupos foram semelhantes em relação a todas as variáveis estudadas, exceto escolaridade, idade gestacional ao parto e procedimentos obstétricos, sendo a escolaridade maior no grupo de feticídio $(p<0,0001)$, a idade gestacional ao parto, menor $(p=0,02)$ e o número de procedimentos obstétricos maior neste grupo $(p=0,01)$. O tempo de indução de parto e as complicações ocorridas também foram semelhantes. Conclusão: a realização do feticídio não altera a evolução clínica das mulheres submetidas à interrupção médica da gestação, podendo ser aplicada nos casos de doença fetal incompativel com a sobrevida perinatal, sem riscos à saúde materna.

PALAVRAS-CHAVE: Aborto terapêutico; Morte fetal; Diagnóstico pré-natal; Complicações na gravidez; Trabalho de parto

Resumo de Tese

\section{Efeitos da hiperprolactinemia sobre a morfologia e os glicosaminoglicanos no útero de camundongas durante as fases do ciclo estral}

Effects of hyperprolactinemia on the morphology and glycosaminoglycan content in the female mouse uterus along the estrous cycle

Autora - Regina Célia Teixeira Gomes

Orientador - Prof. Dr. Manuel de Jesus Simões

Tese apresenteda à Universidade Federal de São Paulo - Escola Paulista de Medicina, para obtenção de Título de Mestre em Ciências, aprovada em 30 de agosto de 2006.

Introdução: a hiperprolactinemia persistente, que resulta em baixa fertilidade, pode acarretar alterações em componentes da matriz extracelular uterina. Objetivos: avaliar os efeitos da hiperprolactinemia (HPrl) na morfologia e nos glicosaminoglicanos (GAGs) da matriz uterina de camundongas durante as fase do ciclo estral. Métodos: oitenta camundongas adultas foram divididas em dois grupos de 40 animais tratados durante 50 dias: controle (CTR), com injeções diárias i.p. de 0,2 $\mathrm{ml}$ de solução salina fisiológica, e experimental (HPrl), com 200 $\mu \mathrm{g}$ ao dia de metoclopramida. Os animais foram sacrificados conforme a fase (proestro, estro, metaestro e diestro). Amostras dos cornos uterinos foram preparadas para microscopia óptica e para processamento bioquímico. Os GAGs foram extraídos por proteólise, caracterizados por eletroforese em gel de agarose e quantificados por densitometria. O ácido hialurônico (AH) foi determinado em ensaio fluorimétrico ELISA-like. Os resultados obtidos foram submetidos à análise estatística utilizando o teste $t$ Student, não pareado. Resultados: os úteros do grupo HPrl apresentaram endométrio mais proliferado e espesso, independente da fase do ciclo estral. As quantidades de condroitim sulfato, dermatam sulfato e ácido hialurônico foram mais elevadas no grupo HPrl nas fases de proestro, estro e diestro; o heparam sulfato apresentou-se significativamente elevado apenas no metaestro. Conclusão: a hiperprolactinemia teve efeitos ciclo estraldependentes sobre a quantidade de GAGs no útero de camundongas. Possivelmente, estas alterações tenham relação com os problemas de infertilidade devidos a esta condição endócrina.

PALAVRAS-CHAVE: Prolactina; Hiperprolactinemia; Glicosaminoglicanos; Útero (Endométrio/Miométrio) 